International Journal of Wireless \& Mobile Networks (IJWMN) Vol. 7, No. 2, April 2015

\title{
Performance Analysis of Adaptive Noise Canceller Employing NlMS Algorithm
}

\author{
Farhana Afroz ${ }^{1}$, Asadul Huq ${ }^{2}$, F. Ahmed ${ }^{3}$ and Kumbesan Sandrasegaran ${ }^{1}$ \\ ${ }^{1}$ Faculty of Engineering and Information Technology, \\ University of Technology, Sydney, Australia \\ ${ }^{2}$ Department of Electrical and Electronic Engineering, \\ University of Dhaka, Bangladesh \\ ${ }^{3}$ Department of Computer Science and Engineering, IUB, Bangladesh
}

\begin{abstract}
In voice communication systems, noise cancellation using adaptive digital filter is a renowned technique for extracting desired speech signal through eliminating noise from the speech signal corrupted by noise. In this paper, the performance of adaptive noise canceller of Finite Impulse Response (FIR) type has been analysed employing NLMS (Normalized Least Mean Square) algorithm. An extensive study has been made to investigate the effects of different parameters, such as number of filter coefficients, number of samples, step size, and input noise level, on the performance of the adaptive noise cancelling system. All the results have been obtained using computer simulations built on MATLAB platform.
\end{abstract}

\section{KEYWORDS}

Adaptive Noise Canceller, NLMS, Number of Samples, Step Size, Filter Coefficients, SNR, NRR

\section{INTRODUCTION}

In communication system, generally different transformational operations are performed on a signal during information transmission [1]. In signal processing, a signal containing useful information is passed through a system (e.g. filter, modulator, adder etc.) to process the signal [2]. In noise cancelling, signal processing is concerned with filtering out the noise from the noisecorrupted signal to recover the signal of interest. The statistics of the noise corrupting a signal is unknown in many situations and changes with time. Moreover, the power of noise may be greater than the power of the desired signal being transmitted. In these circumstances, conventional nonadaptive digital filters may not show satisfactory performances and the noise cancelling should be an adaptive process i.e. the noise canceller should be capable to adapt itself with changing environments. Adaptive noise cancellation is an operation of suppressing background noise from useful signals that is controlled in an adaptive manner in order to obtain improved SNR (Signal to Noise Ratio) at the receiving end [3, 4]. In general, an adaptive noise canceling system consists of an adaptive filter, two sensing systems and a subtracting unit. The primary concept of an adaptive noise cancelling algorithm is to input the noise-corrupted signal to the digital filter which in turn processes that noisy signal to remove the noise while leaving the useful signal unaffected [5]. The adaptive filter coefficients get adjusted automatically according to the changes of the input signal

DOI : 10.5121/ijwmn.2015.7204 
International Journal of Wireless \& Mobile Networks (IJWMN) Vol. 7, No. 2, April 2015

characteristics [6]. This paper provides a study of the performance of an adaptive noise canceller employing NLMS (Normalized Least Mean Square) algorithm. The performance of the system is analysed while varying a range of parameters such as step size, number of filter coefficients, input noise level and number of samples.

The rest of this paper is organized as follows. A short literature review of related work is presented in Section 2. An adaptive noise cancelling system is illustrated in Section 3 followed by descriptions of adaptive algorithms in Section 4. Section 5 demonstrates the simulation parameters and results. Finally, Section 6 concludes the paper.

\section{RELATED WORK}

The field of adaptive filter's application is highly enriched with a very big volume of literature. In this section, attempts will be made to report some of these works.

The initial works on adaptive filtering applications can be traced back in the 1950s. In 1959 the Least Mean Square (LMS) algorithm was invented by Widrow and Hoff in their study of a pattern recognition scheme known as the adaptive linear threshold logic element [7]. The LMS algorithm and the idea of stochastic approximation method (developed by Robbins and Monro in [8]) in statistics for solving sequential parameter estimation problems are closely related with each other. The basic difference between them is that in case of LMS scheme, the algorithm parameter, (i.e. step size) which is used to adjust the correction applied to the tap weight vector from one iteration to the next, is held constant, whereas in stochastic approximation methods the step size is maintained to be inversely proportional to time $\mathrm{n}$ [4]. Godard in 1974, contributed to the development of adaptive filtering algorithms. Kalman filter theory was utilized in his work to devise a novel class of adaptive filtering schemes for achieving fast convergence of the transversal filter's tap weights to their optimum values [9]. A comparison of the performances of RLSL (Recursive Least Squares Lattice) and normalized step-size SGL (Stochastic Gradient Lattice) schemes to that of the LMS transversal scheme for cancelling sinusoidal interferences was made in [10]. The experimental results showed that the adaptive lattice filters is more advantageous than LMS transversal filter, which makes them more preferable adaptive noise canceller (ANC) filter structure if the increased computational cost can be accepted. A comparative study of the performances of adaptive filtering algorithms was showed in [11]. In this work, the performances of TV-LMS (time-varying LMS), LMS and RLS (Recursive Least Square) algorithms were studied and compared in terms of algorithm execution time, required filter order and MSE (Mean Square Error). A study of mean-square performance of adaptive filters employing averaging theory was presented in [12]. This paper studied mean-square error and mean-square deviation performance of adaptive filters along with the transient behavior of the corresponding partially averaged systems. In [13], a simple neural network namely Adaline was utilized as adaptive filter for cancelling engine noise in a car. The experimental results in [13] showed that the SNR gets improved after passing through the noise cancelling system. A new class of nonlinear adaptive filter namely ANFF (Adaptive Neural Fuzzy Filter) with adaptive fuzziness parameters and adaptive learning ability was developed in [14]. In addition, an adaptive noise canceller was simulated to verify the efficiency of the newly developed ANFF. A new approach in noise cancellation was proposed in [15] in which two adaptive algorithms namely FAP (Fast Affine Projection) and FEDS (Fast Euclidean Direction Search) algorithms are employed for cancelling 
International Journal of Wireless \& Mobile Networks (IJWMN) Vol. 7, No. 2, April 2015

noise in speech signal. In addition, the obtained results are compared with the results obtained with RLS, LMS, NLMS (Normalized LMS) and AP (Affine Projection) algorithms. A DSP-based oversampling adaptive noise canceller employing RLS algorithm for reducing background noise for mobile phones was presented as well as the performance of the system was analysed in [16]. A novel adaptive noise cancelling scheme having low computation complexity was proposed in [17] for cancelling different kinds of noise in ECG signal. In [18], an adaptive scheme namely NTVLMS (New Time Varying LMS) has been proposed and the performance of the proposed algorithm is compared with other well-known adaptive schemes such as NLMS, LMS, NVSSLMS (New Variable step size LMS), TVLMS and RVSSLMS (Robust Variable step size LMS).

\section{Adaptive Noise Canceller}

Adaptive noise canceller is utilized to eliminate background noise from useful signals where a signal of interest becomes submerged in noise. One basic element of an adaptive noise cancelling system is adaptive filter. A digital filter having self-adjusting characteristics is known as adaptive filter. An adaptive filter gets adjusted automatically to the changes occurred in its inputs [19]. The coefficients of the adaptive filter are not fixed, rather these can be changed to optimize some measure of the filter performance.

In many applications, a frequently encountered problem is the corruption of desired signal by noise or other unwanted signals. Conventional linear filters, in which the filter coefficients are fixed, generally can be utilized to extract the signal of interest in some situations when the frequency bands occupied by the noise and signal are fixed and not spectrally overlapped with each other. However, many situations exist when there is a spectral overlapping between the desired signal and unwanted signal or the frequency band occupied by the noise is not known or changes with time. The filter coefficients can not be defined in advance in such situations and it must be a variable i.e. the filter characteristics need to be adjusted or altered intelligently according to the changes in its input signal characteristics in order to optimize its performance.

Fig. 1 shows a model of adaptive noise cancelling system. As seen in figure, an adaptive noise canceller consists of two inputs (known as primary input and reference input) and an adaptive filter. The noise-corrupted signal $\left(y_{k}=s_{k}+n_{k}\right)$ is applied as primary input. Reference input is the noise, $\overline{x_{k}}$ which is correlated with the main input, $x_{k}$ in some way but uncorrelated with the signal, $s_{k}$. The noise reference input is applied to the adaptive filter and an output, $\widehat{n}_{\mathrm{k}}$ is estimated which is a close replica of $n_{k}$ as much as possible. The adaptive filter readapts itself incessantly so that the error between $n_{k}$ and $\widehat{n}_{\mathrm{k}}$ is minimized during this process. Finally, the recovered signal is obtained by subtracting the estimated noise, $\widehat{n}_{\mathrm{k}}$ from the primary input.

As shown in Fig. 1, the adaptive noise cancelling system model contains a band limit noise filter, a noise reference filter and an adaptive filter. The band limit noise FIR (Finite Impulse Response) filter is used to make the model more realistic to the environment. Noise is not always white in nature. This filter allows passing a selected portion of the white noise spectra. As a result, a colored noise $\left(n_{k}\right)$ is obtained in the output section. The output of the noise reference filter $\left(\overline{x_{k}}\right)$ is simultaneously fed to the digital FIR filter section and the adaptive weight control mechanism unit. Thus, a correlative behavior can be established between the noisy component of input and 
International Journal of Wireless \& Mobile Networks (IJWMN) Vol. 7, No. 2, April 2015

reference noise. The adaptive filter has two parts: an FIR digital filter with adaptable tap weights or coefficients, and an adaptive algorithm through which filter tap weights can be adjusted or modified so that error can be minimized [3].

The desired output of the adaptive noise canceller is given by

$$
\begin{aligned}
\hat{s}_{\mathrm{k}} & =\mathrm{e}_{\mathrm{k}}=\mathrm{y}_{\mathrm{k}}-\widehat{n}_{\mathrm{k}} \\
& =s_{k}+n_{k}-\widehat{n}_{\mathrm{k}}
\end{aligned}
$$

where, $s_{k}, n_{k}, \mathrm{y}_{\mathrm{k}}$ and $\mathrm{e}_{\mathrm{k}}$ are termed as the useful signal, the band-limited noise, the noisecorrupted signal and the error signal respectively.

The FIR filter output is [20]

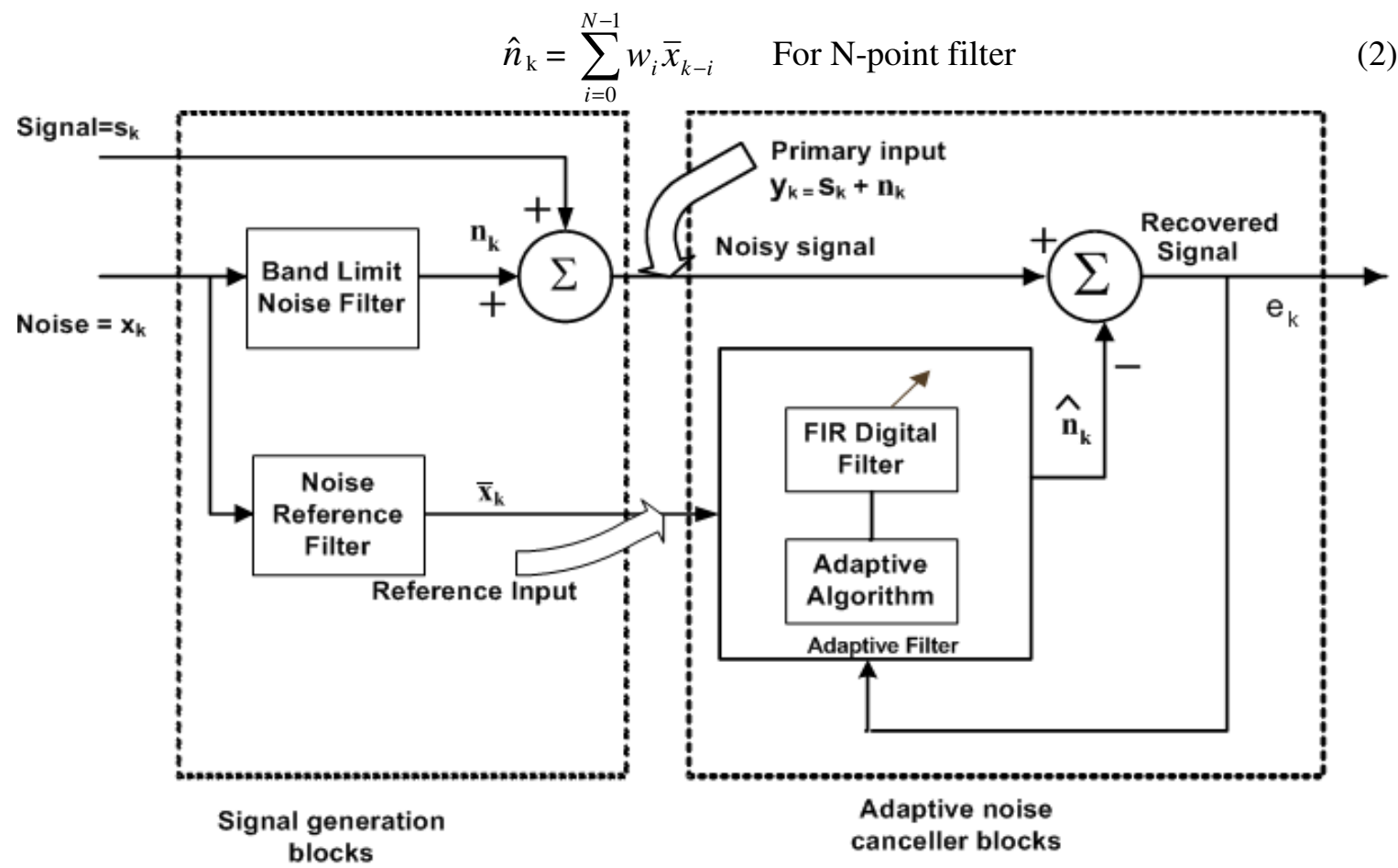

Figure 1: A Model of Adaptive Noise Canceller

Where, $w_{i}=\left[w_{o}, w_{1}, \ldots w_{N-1}\right]$ are the adjustable filter coefficients [21] and, $\bar{x}_{k}$ and $\hat{n}_{k}$ are the input and output of the filter respectively.

\section{Adaptive Algorithm}

Many adaptive algorithms have been proposed for implementing adaptive filter theory. In this work, we have applied NLMS (Normalized Least Mean Square) algorithm for studying adaptive noise cancelling system's performance. The normalized LMS (NLMS) algorithm may be viewed 
International Journal of Wireless \& Mobile Networks (IJWMN) Vol. 7, No. 2, April 2015

as a special implementation of the LMS algorithm. In the following sub-sections, LMS and NLMS algorithms are illustrated.

\subsection{Least Mean Square (LMS)}

Widrow and Hoff first proposed the LMS algorithm in 1960. It is based on the steepest descent algorithm. This algorithm modifies the filter coefficients in such a way that $e_{k}$ gets minimized in the mean-square sense. It is a sequential mechanism which can be employed to adjust the filter tap weights by continuously observing its input and desired output.

If the filter input vector is $x(n)$ and the desired output vector is $d(n)$, then the filter output, $y(n)$ and estimated error signal, e(n) can be written as equation (3) and (4) respectively [22].

$$
\begin{aligned}
& y(n)=w^{T}(n) x(n) \\
& e(n)=d(n)-y(n)
\end{aligned}
$$

where, $\mathrm{w}(\mathrm{n})$ is the filter tap weight vector.

For LMS algorithm, at each iteration, the weight vector is updated by a small amount according to the following equation [22]:

$$
w(n+1)=w(n)+2 \mu e(n) x(n)
$$

where, $\mu$ is called the algorithm step size. $\mu$ is a convergence factor, whose value decides by which amount the tap weight vector will be changed at each iteration.

\subsection{Normalized Least Mean Square (NLMS)}

The NLMS (Normalized Least Mean Square) scheme can be seen as a special implementation of the Least Mean Square (LMS) algorithm which considers the variations in the signal level at the input of the filter and chooses a normalized step-size parameter that yields in a stable adaptation algorithm having fast convergence rate. In NLMS algorithm the step size parameter $\mu$ is normalized to $\mu_{\mathrm{n}}$ and the tap weights are updated according to the following equation [22]:

Here,

$$
w(n+1)=w(n)+\frac{\mu e(n) x(n)}{x^{T}(n) x(n)+\varphi}
$$

$\mu_{n}=\frac{\mu}{x^{T}(n) x(n)+\varphi}$

where, $\varphi$ is a small constant, used to avoid the numerical instability of algorithm that may arise, and $x(n), e(n), w(n)$ and $\mu$ represent the filter input vector, the estimated error signal, the filter tap weight vector and the step size respectively.

Normalized LMS algorithm can be viewed as an LMS algorithm with a time-varying step size parameter. In addition, normalized LMS algorithm leads to faster rate of convergence as compared with that of the standard LMS algorithm both for correlated and uncorrelated input data [23]. 


\section{Simulations AND ReSUlts}

The performance evaluation of adaptive noise canceller employing NLMS algorithm is reported in this section. The performance is analysed with varying some parameters such as step size, number of filter coefficients, input noise level and number of samples. All the results are obtained using computer simulations built on MATLAB platform. A recorded speech signal (shown in Fig. 2) with following characteristics has been taken into consideration to study the system's performance.

$\begin{array}{ll}\text { Number of samples } & : 24000 \\ \text { Bit rate } & : 64 \mathrm{kbps} \\ \text { Audio sample size } & : 8 \mathrm{bit} \\ \text { Audio sampling rate } & : 8000 \mathrm{~Hz} \\ \text { Audio format } & : \text { PCM } \\ \text { Channels } & : 1 \text { (mono) }\end{array}$

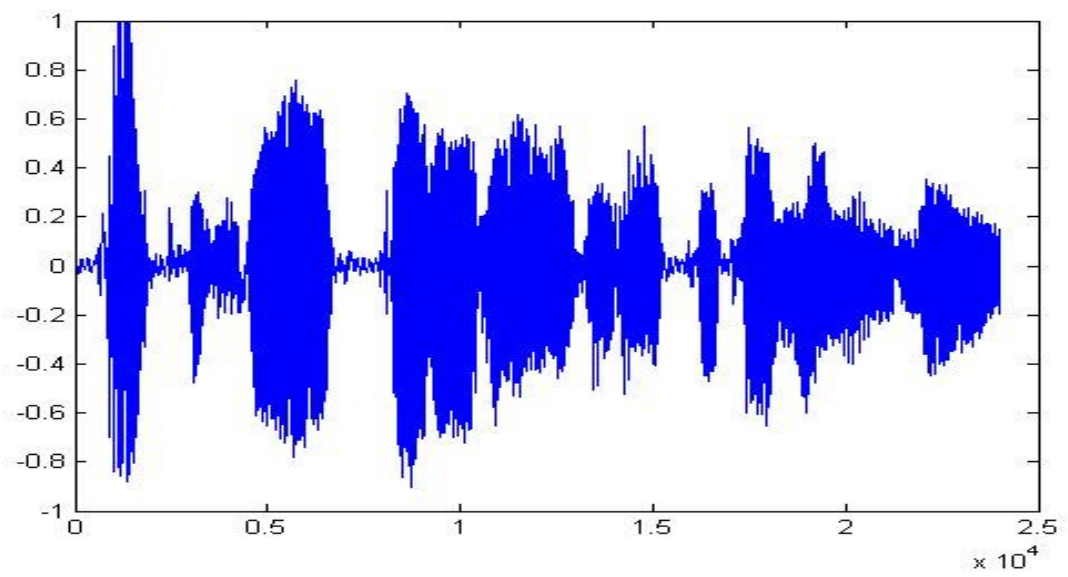

Figure 2: A speech signal

\subsection{Effects of number of filter coefficients}

Twenty observations were made to evaluate the variations of the system's performance with the number of filter coefficients. The performance of the system is measured by calculating Noise Reduction Ratio (in $\mathrm{dB}$ ). Noise-reduction-ratio (NRR) is the ratio of noise power to the error power [24].

$$
\begin{gathered}
\text { NRR }=\frac{\text { Noise power }}{\text { Error power }} \\
N R R(d B)=10 \log _{10}(N R R)
\end{gathered}
$$

The variations of NRR with changing number of filter tap weights are tabulated in Table 1. It is seen in Fig. 3 that on an average the Noise Reduction Ratio of the system tends to decrease while 
International Journal of Wireless \& Mobile Networks (IJWMN) Vol. 7, No. 2, April 2015 the number of filter coefficients is increased. It is also observed that NRR reaches its maximum value of $29.9992 \mathrm{~dB}$ when the number of filter coefficients is 7 after which it declines with an increase of number of filter taps.

Table 1: Effects of number of filter coefficients

\begin{tabular}{|c|c|}
\hline \multicolumn{2}{|c|}{$\begin{array}{l}\text { Simulation parameters } \\
\text { Number of samples: } 20000 \\
\text { Noise Power: }-16.8004 \\
\text { Step Size : } 0.15 \\
\text { Frequency range of colored noise: } 1200-2000 \mathrm{~Hz}\end{array}$} \\
\hline Number of filter coefficients & Noise Reduction Ratio (NRR) in dB \\
\hline 3 & 27.0656 \\
\hline 4 & 28.7159 \\
\hline 5 & 28.9636 \\
\hline 6 & 29.1950 \\
\hline 7 & 29.9992 \\
\hline 10 & 29.1705 \\
\hline 12 & 29.4239 \\
\hline 14 & 29.1195 \\
\hline 16 & 29.4878 \\
\hline 18 & 28.6723 \\
\hline 20 & 29.1335 \\
\hline 23 & 29.0453 \\
\hline 25 & 28.8233 \\
\hline 28 & 28.8344 \\
\hline 30 & 27.9594 \\
\hline 32 & 28.1655 \\
\hline 50 & 27.2856 \\
\hline 64 & 27.5548 \\
\hline 70 & 24.6840 \\
\hline
\end{tabular}




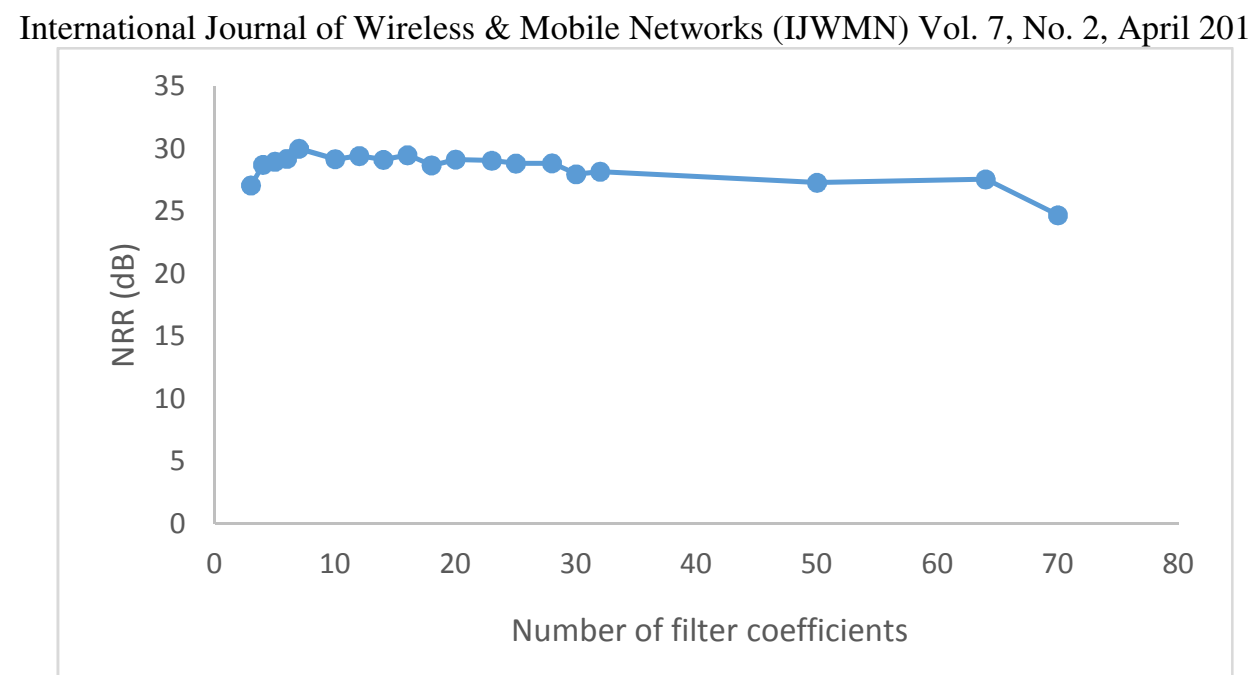

Figure 3: Variation of NRR (in $\mathrm{dB}$ ) with number of tap weights

\subsection{Effects of step size}

The effects of step size (adaptive algorithm parameter) on the performance of the system is evaluated in this subsection. The step size is increased from 0.01 to 0.2 and the system's performance corresponding to respective step size is measured in terms of Noise Reduction Ratio (NRR). The simulation parameters and the results obtained are tabulated in Table 2. A graphical representation of tabular data is shown in Fig. 4. It is observed from Fig. 4 that above a particular value of step size (0.03), the NRR gradually declines with increasing step size. Below that value, NRR gradually increases with the increase in step size. The optimum step size [25] (at which the best noise reduction is seen) is 0.03 for the given simulation parameters.

Table 2: Effects of step size

\begin{tabular}{|c|c|}
\hline \multicolumn{2}{|c|}{$\begin{array}{l}\text { Simulation parameters } \\
\text { Number of samples: } 15000 \\
\text { Noise Power: }-16.8004 \\
\text { No. of filter coefficients: } 32 \\
\text { Frequency range of colored noise: } 1200-2000 \mathrm{~Hz}\end{array}$} \\
\hline Step Size & $\begin{array}{l}\text { Noise Reduction Ratio } \\
\text { (in dB) }\end{array}$ \\
\hline .01 & 26.2926 \\
\hline .02 & 26.6716 \\
\hline .03 & 27.1593 \\
\hline .04 & 26.9859 \\
\hline .05 & 26.0503 \\
\hline .06 & 25.4877 \\
\hline .07 & 24.9614 \\
\hline .08 & 24.8289 \\
\hline .09 & 23.4080 \\
\hline
\end{tabular}


International Journal of Wireless \& Mobile Networks (IJWMN) Vol. 7, No. 2, April 2015

\begin{tabular}{|c|c|}
\hline 0.1 & 23.9092 \\
\hline 0.15 & 21.6609 \\
\hline 0.2 & 20.3737 \\
\hline
\end{tabular}

The pictorial representation of noisy signal i.e. the original speech signal contaminated with color noise (upper part), and the recovered speech signal at optimum step size (lower part) are shown in Fig. 5. It can be observed that the recovered speech signal is identical to the original speech signal.

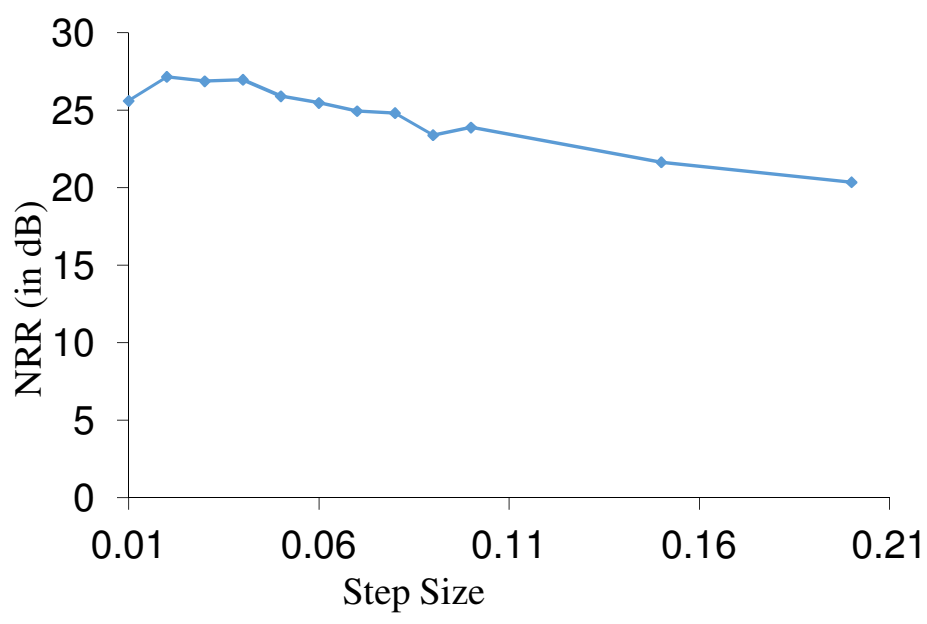

Figure 4: Variations of NRR with step size 
International Journal of Wireless \& Mobile Networks (IJWMN) Vol. 7, No. 2, April 2015
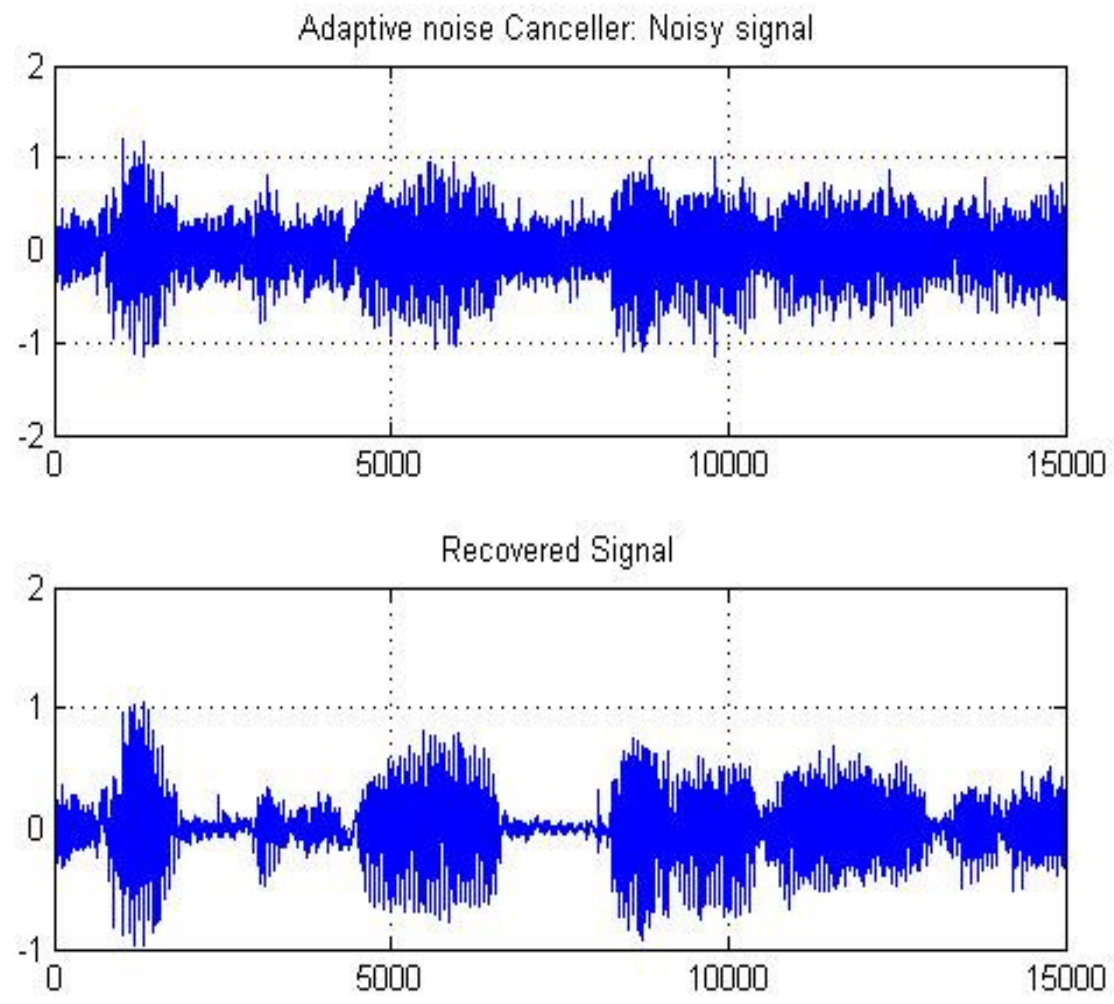

Figure 5: Noisy signal and recovered signal for step size $=0.03$

Table 3: Optimum step size with different number of samples

\section{Simulation parameters}

Noise Power: -16.8004

No. of filter coefficients: 32

Frequency range of colored noise: $1200-2000 \mathrm{~Hz}$

\begin{tabular}{|c|c|c|}
\hline $\begin{array}{c}\text { Number of } \\
\text { Samples }\end{array}$ & Optimum Step Size & NRR in dB \\
\hline 20000 & 0.02 & 28.4713 \\
\hline 15000 & 0.03 & 27.1593 \\
\hline 10000 & 0.03 & 26.9450 \\
\hline
\end{tabular}

It is also observed from the Table 3 that the optimum step size is affected by the number of samples of the signal while number of filter coefficients and noise power are kept identical in each case. It is also seen that if the number of samples is decreased under identical simulation parameters, the NRR of the system becomes worse. 


\subsection{Effects of the number of samples}

The effect of number of samples on the performance of the adaptive noise cancelling system is studied here. The performance is measured in terms of Noise Reduction Ratio (NRR). Results are tabulated in following table (Table 4) and graphically represented in Fig. 6.

Table 4: Effects of number of samples

\begin{tabular}{|c|c|}
\hline $\begin{array}{l}\text { Simulation parameters } \\
\text { Step size: } 0.15 \\
\text { Noise Power: }-16.8004 \\
\text { No. of filter coefficients: } 32\end{array}$ & \\
\hline Number of samples & Noise Reduction Ratio (in dB) \\
\hline 4000 & 23.0838 \\
\hline 6000 & 21.6214 \\
\hline 8000 & 22.3855 \\
\hline 10000 & 21.9996 \\
\hline 12000 & 21.8909 \\
\hline 14000 & 21.7266 \\
\hline 18000 & 22.2465 \\
\hline 20000 & 22.3013 \\
\hline 22000 & 22.3687 \\
\hline 24000 & 22.6737 \\
\hline
\end{tabular}

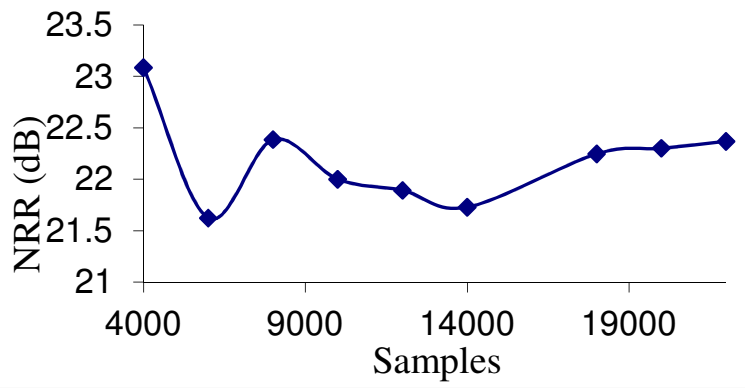

Figure 6: Noise Reduction Ratio (in $\mathrm{dB}$ ) versus number of samples

To study the system's performance, several observations have been made by increasing the number of samples from 4000 up to 24000 and measuring the corresponding NRR for respective number of samples. It is seen from the Fig. 6 that after some initial fluctuations the NRR gradually becomes stable as the number of samples is increased. The initial fluctuation might be due to the inadequate number of samples employed for which the filter could not adapt to sudden changes in the signal. However, it is somewhat difficult to make a firm conclusion regarding this trend at this stage. More research works are needed to predict the correct reasons regarding this issue. 


\subsection{Effects of input SNR (Signal to Nose Ratio)}

The impact of input Signal to Noise Ratio (the ratio of signal power to noise power) on the performance of the system is analysed in this part. The performance is measured in terms of NRR. The results obtained under the simulation parameters are given in Table 5 and graphically represented in Fig. 7.

Table 5: Effects of input SNR on NRR

\begin{tabular}{|c|c|}
\hline $\begin{array}{l}\text { Simulation parameters } \\
\text { Number of samples: } 10000 \\
\text { Step size: } 0.03 \\
\text { No. of filter coefficients: } 32\end{array}$ & \\
\hline Input SNR in dB & Noise Reduction Ratio (NRR) in dB \\
\hline 3 & 26.0640 \\
\hline 4 & 26.3715 \\
\hline 5 & 27.9538 \\
\hline 6 & 27.7578 \\
\hline 7 & 27.7716 \\
\hline 8 & 27.6028 \\
\hline 9 & 27.4978 \\
\hline 10 & 28.0481 \\
\hline 11 & 30.0222 \\
\hline 12 & 28.7785 \\
\hline
\end{tabular}

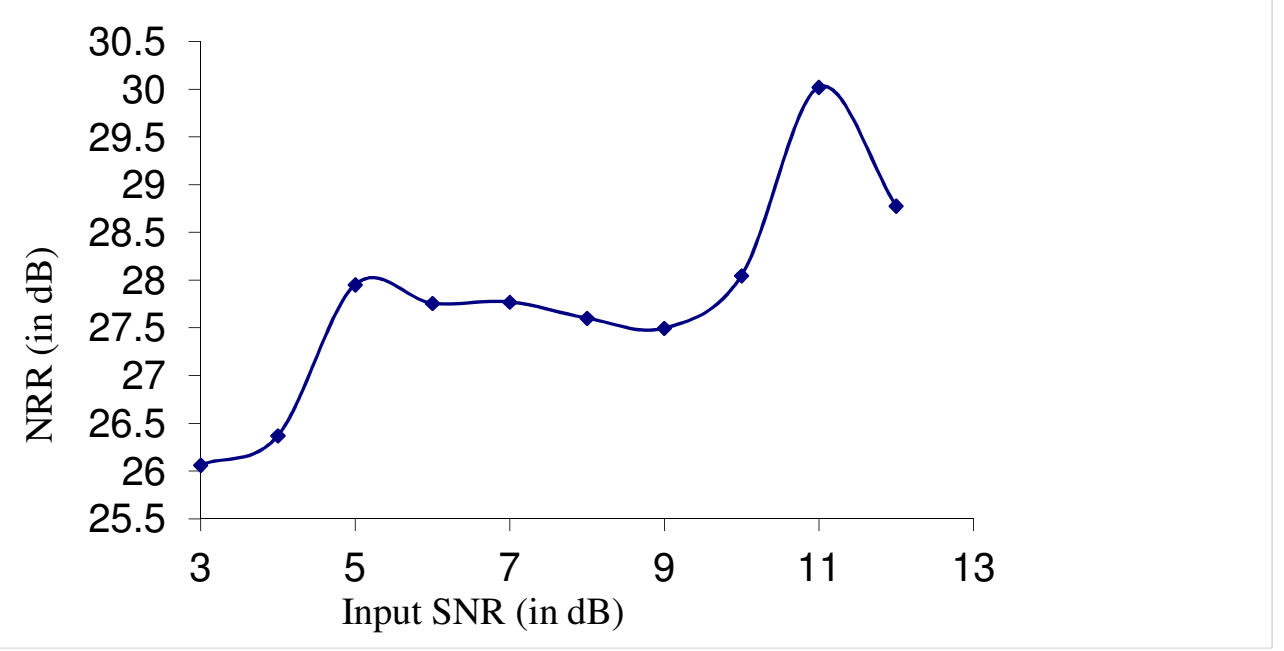

Figure 7: Input SNR versus NRR

As seen from the table, ten observations were made to evaluate the effects of input SNR on the system's performance. The input SNR is increased from $3 \mathrm{~dB}$ to $12 \mathrm{~dB}$ and the respective NRR is 
International Journal of Wireless \& Mobile Networks (IJWMN) Vol. 7, No. 2, April 2015

calculated for each SNR value. It is seen from the Fig. 7 that if the SNR is gradually increased the NRR is gradually increased till the input SNR is $5 \mathrm{~dB}$ followed by a drop of NRR on average with increasing SNR up to $9 \mathrm{~dB}$. Subsequently, NRR again starts rising with increasing SNR up to 12 $\mathrm{dB}$ after which it declines when the SNR is further increased by $1 \mathrm{~dB}$. It was also observed that the system showed best performance at an input SNR of $11 \mathrm{~dB}$ and worst performance at $3 \mathrm{~dB}$ input SNR.

\section{Conclusion}

In this paper, the performance of adaptive noise canceller using NLMS algorithm has been evaluated with varying different parameters of the system. The adaptation capability of the system to any input noise situation as well as the effects of step size, number of filter coefficients, number of samples and input noise level on the performance of the system are thoroughly studied considering a speech signal as useful signal. It is evident that individually each of these parameters has an optimum value at which the adaptive noise canceller showed best performance. Our future work includes, making a comparison of the performance of adaptive noise cancelling system employing RLS (Recursive Least Square) and LMS (Least Mean Square) algorithms through computer simulations and then double check the obtained results by performing the real-time experiments using DSP hardware.

\section{REFERENCES}

[1] Khanna, V. K. (2009), Digital Signal Processing, S. Chand and Company Limited, New Delhi.

[2] C. Durai, R. B. (2005), Digital Signal Processing, Laxmi Publications LTD, New Delhi.

[3] Ifeachor, E. C. and Jervis, B. W. (1993), Digital Signal Processing, Addison-Wesley Publishing Company.

[4] Haykin, S. (1996), Adaptive Filter Theory, 3rd edition, Prentice-Hall International Inc.

[5] Widrow, B., Glover, J.R., Mccool, J.M., Kaunitz, J., Williams, C.S., Hearn, R.H., Zeidler, J.R., Eugene Dong, Jr. and Goodlin, R.C. (1975), "Adaptive noise cancelling: Principles and Applications", Proceedings of the IEEE, Vol.63(12), pp.1692-1716.

[6] Ramli, R.M., Ali O. Noor, A.O. and Samad, S.A. (2012), "A Review of Adaptive Line Enhancers for Noise Cancellation", Australian Journal of Basic and Applied Sciences, 6(6): 337-352.

[7] Widrow, B. and Hoff, M.E. (1960), "Adaptive switching circuits", Proceedings of WESCON Convention Record, part 4, pp.96-104.

[8] Robbins, H. and Monro, S. (1951), "A stochastic approximation method", The Annals of Mathematical Statistics, Vol.22(3), pp.400-407.

[9] Godard, D. (1974), "Channel equalization using a Kalman filter for fast data transmission", IBM journal of Research and Development, Vol.18(3), pp.267-273.

[10] North, R.C., Zeidler, J.R., Albert, T.R. and Ku W.H. (1992), "Comparison of Adaptive Lattice Filters to LMS Transversal filters for Sinusoidal Cancellation", IEEE International Conference on Acoustics, Speech, and Signal Processing, Vol. 4, pp. 33-36.

[11] Lau, Y.S. Hossain, Z.M. and Harris, R. (2003), "Performance of Adaptive Filtering Algorithms: A Comparative Study", Proceedings of the Australian Telecommunications, Networks and Applications Conference (ATNAC), Melbourne.

[12] Shin, H.C., Sayed, A.H. and Song, W.J. (2004), "Mean Square Performance of Adaptive Filters using Averaging Theory", IEEE Conference on Signals, Systems and Computers, Vol. 1, pp.229-234. 
International Journal of Wireless \& Mobile Networks (IJWMN) Vol. 7, No. 2, April 2015

[13] Stella, M., Begusic, D. and Russo, M. (2006), "Adaptive noise cancellation based on neural network", International Conference on Software in Telecommunications and Computer Networks, pp.306-309.

[14] Golpayegani, G.N. and Jafari, A.H. (2009), "Improved adaptive neural fuzzy filter and its application in noise cancellation", 3rd International Conference on Bioinformatics and Biomedical Engineering, pp.1-7.

[15] Hadei, S.A. and Lotfizad, M. (2010), "A family of adaptive filter algorithms in noise cancellation for speech enhancement", International Journal of Computer and Electrical Engineering, Vol.2, No.2.

[16] Tavares Rayes, J.A., Escamilla Hernandez, E. and Sanchez Garcia, J.C. (2012), "DSP-based oversampling adaptive noise canceller for background noise reduction for mobile phones", 22nd International Conference on Electrical Communications and Computers, pp.327-332.

[17] Mohammed, J.R. (2014), "Low complexity adaptive noise canceller for mobile phones based remote health monitoring", International Journal of Electrical and Computer Engineering, Vol.4, No. 3, pp.422-432.

[18] Siddappaji and Sudha, K.L. (2015), "Performance analysis of new time varying LMS (NTVLMS) adaptive filtering algorithm in noise cancellation system", International Conference on Communication, Information \& Computing Technology, pp. 1-6.

[19] Yadev, C.S., Krishna, B.A. (2014), "Study of Different Adaptive Filter Algorithms for Noise Cancellation in Real-Time Environment", International Journal of Computer Applications, Volume 96- No.10.

[20] Proakis, J.G. and Manolakis, D.G. (1992), Digital Signal Processing: Principles, Algorithms, and Applications, New York: Macmillan; Toronto: Maxwell Macmillan Canada.

[21] Sazzad, Z.M.P., Alam, M. S., Moslehuddin, Huq, A. and Ahmed, F. (1999), "Design aspects of window-based digital FIR filters", Dhaka University Journal of Science, 47(1): 5-13.

[22] Farhang-Boroujeny, B. (2013), Adaptive Filters: Theory and Applications, John Wiley \& Sons.

[23] Perez-Meana, H. (2007), Advances in Audio and Speech Signal Processing: Technologies and Applications, Idea Group Inc. (IGI).

[24] Rubak, P. Johansen, L.G. \& Hermansen, K. (1996), "Adaptive Noise Canceling in Headsets", Proceedings of Nordic Acoustical Meeting, NAM'96, Helsinki, Finland, pp. 99-104.

[25] Huq, A., Zhiqiang, M.A. and Kenji, N. (1993), A Method for determining the Optimum Tap Combination in Pole Zero Adaptive Filters, Technical Report of IECE. 